\title{
Horndeski Genesis: strong coupling and absence thereof
}

\author{
Yulia Ageeva ${ }^{1,2, *}$, Oleg Evseev ${ }^{1,2, * *}$, Oleg Melichev ${ }^{1,2, * * *}$, and Valery Rubakov ${ }^{1,2, * * * *}$ \\ ${ }^{1}$ Institute for Nuclear Research of the Russian Academy of Sciences, 60th October Anniversary \\ Prospect 7a, 117312 Moscow, Russia \\ ${ }^{2}$ Department of Particle Physics and Cosmology, Physics Faculty, M.V. Lomonosov Moscow State \\ University, Vorobjevy Gory, 119991, Moscow, Russia
}

\begin{abstract}
We consider Genesis in Horndeski theory as alternative to or completion of inflationary scenario. One of the options free of instabilities at all comological epochs is putative strong coupling regime at the early Genesis stage. We address this issue to see whether classical field theory description of the background evolution at this early stage is consistent, nevertheless. We argue that, indeed, despite naive strong coupling, classical analysis is legitimate in a certain range of parameters. This opens up a posibility of a novel scenario for the beginning of the cosmological expansion.
\end{abstract}

\section{Introduction}

Genesis [1]-[7] is an interesting alternative to or completion of inflationary cosmology. In this scenario the Universe starts its evolution from asymptotically flat space-time at infinitely negative time. During the time evolution the energy density, scale factor and Hubble rate grow. At some moment of time the Genesis regime is assumed to terminate, and conventional hot (or inflationary) epoch begins.

Genesis requires the violation of the Null Energy Condition (NEC) (for a review see, e.g., Ref.[8]). To violate the NEC in a healthy way, one needs unusual matter. In a general non-canonical scalar field theory whose Lagrangian depends on $\phi$ and its first derivatives, the NEC can be violated. However, NEC-violating cosmological solutions are unstable because the curvature perturbation has either wrong sign kinetic term [9], [10] or gradient instability or both. Healthy NEC violation can be obtained in generalized Galileon/Horndeski theory [11] [18]. This is the most general scalar-tensor theory with second-order field equations. Such a property is instrumental for avoiding Ostrogradski instabilities, i.e. the ghost-like DOF that are usually associated with higher-order time derivatives. In the original Genesis model and in its versions, the initial super-accelerating stage can occur without these instabilities.

There is an issue in Horndeski Genesis models, however. In most cases, spatially flat Genesis solutions are plagued with gradient instabilities occuring sooner or later in the cosmological evolution [19-22]. This has been formulated as the "no-go theorem". One of possible ways out is to consider models which are, at least naively, strongly coupled in the asymptotic past (and/or asymptotic future, the case that can be studied along the lines of this

\footnotetext{
*e-mail: y.a.ageeva2604@gmail.com

**e-mail: oa.evseev@physics.msu.ru

***e-mail: olegmelichev@gmail.com

****e-mail: rubakov@ms2.inr.ac.ru
} 
work) $[20,23,25]$. In these models, the coefficients in quadratic action for perturbations about the classical solution tend to zero as $t \rightarrow-\infty$, which, indeed, implies that the strong coupling energy scale also tends to zero.

In this paper we point out that this property does not necessarily mean that one cannot use classical field theory for describing the cosmological evolution at early times. Indeed, the time scale of the classical evolution tends to infinity, and hence its inverse, the classical energy scale tends to zero, as $t \rightarrow-\infty$. So, to see whether or not the classical field theory treatment is legitimate, one has to figure out the actual strong coupling energy scale and compare it with inverse time scale of the classical background evolution. The classical analysis of the background is consistent, provided that the former energy scale much exceeds the latter. In this paper we consider a simple class of Horndeski Genesis models with strong coupling at early times, and study scalar perturbations in the asymptotics $t \rightarrow-\infty$. We derive the conditions ensuring that the classical energy scale is much lower than the strong coupling scale in the scalar sector. We find that these conditions can indeed be satisfied in a certain range of parameters in the Lagrangian, i.e., it is possible to avoid strong coupling regime for Genesis stage at least as far as the scalar sector is concerned, in the sense that the classical treatment of the background evolution is consistent at early times. We argue that tensor and tensor-scalar sectors may leave this result unmodified.

This paper is organized as follows. In Section 2 we introduce the model and discuss its early-time asymptotics that enable one to avoid the no-go theorem of Ref. [20]. In Section 3 we discuss strong coupling issue in detail and find a region of the parameter space in which the classical description of Genesis is legitimate despite the low strong coupling energy scale. We conclude in Section 4.

\section{Generalities}

\subsection{The model}

If one uses general relativity to describe gravity, then an important characteristic is the null energy condition (NEC) for the matter energy-momentum tensor $T_{\mu \nu}$, which reads $T_{\mu \nu} k^{\mu} k^{\nu} \geq 0$ for every null vector $k^{\mu}$. Once the NEC holds in the cosmological context, then (assuming flat spatial sections) it follows from the Einstein equations that $d H / d t \leq 0$, where $H$ is the Hubble parameter. This implies that there is a singularity in the past of the expanding universe. Therefore, one either modifies gravity or violates the NEC to build non-singular cosmology.

A candidate for NEC violating theory is the generalized Galileon scalar field coupled to gravity [1]-[7]. The most general form of Langangian which leads to second-order field equations was obtained by Horndeski in [11]. It is sufficient for our purposes to consider a subclass of Horndeski Lagrangians instead of full one,

$$
\begin{aligned}
\mathcal{L} & =G_{2}(\phi, X)-G_{3}(\phi, X) \square \phi+G_{4}(\phi) R, \\
X & =-\frac{1}{2} g^{\mu v} \partial_{\mu} \phi \partial_{\nu} \phi,
\end{aligned}
$$

where $R$ is a Ricci scalar and $\square \phi=g^{\mu \nu} \nabla_{\mu} \nabla_{\nu} \phi$. Metric signature is $(-,+,+,+)$.

Let us rewrite this Lagrangian (1) in terms of ADM variables, to make contact with Ref. [20]:

$$
\mathcal{L}=A_{2}(t, N)+A_{3}(t, N) K++A_{4}\left(K^{2}-K_{i j}^{2}\right)+B_{4}(t, N) R^{(3)},
$$

where $\phi=$ const are taken to be equal time hypersurfaces, and $K_{i j}, R_{i j}^{(3)}$ are the extrinsic curvature and the Ricci tensor of the spatial slices. There is one-to-one correspondence between 
the variables $\phi$ and $X$ in the covariant Lagrangian and time variable $t$ and lapse function $N$ in the ADM formalism. The following expressions convert one formalism to another [26], [27]

$$
\begin{aligned}
& G_{2}=A_{2}-2 X F_{\phi}, \\
& G_{3}=-2 X F_{X}-F, \\
& G_{4}=B_{4},
\end{aligned}
$$

where $F(\phi, X)$ is an auxiliary function, such that

$$
F_{X}=-\frac{A_{3}}{(2 X)^{\frac{3}{2}}}-\frac{B_{4 \phi}}{X},
$$

and the following gauge is fixed with $Y_{0}=$ const:

$$
e^{-\phi}=-\sqrt{2 Y_{0}} t \text {. }
$$

\subsection{Avoiding the no-go theorem}

A subclass of Lagrangians in which the no-go theorem can be avoided was given in Ref. [20]:

$$
\begin{aligned}
& A_{2}=M_{P l}^{4} f^{-2(\alpha+1)-\delta} a_{2}(N), \\
& A_{3}=M_{P l}^{3} f^{-2 \alpha-1-\delta} a_{3}(N), \\
& A_{4}=-B_{4}=-M_{P l} f^{-2 \alpha},
\end{aligned}
$$

where $M_{P l}$ is Planck mass, $\alpha$ and $\delta$ are constant parameters satisfying

$$
2 \alpha>1+\delta, \quad \delta>0,
$$

and $f(t)$ is some function of time, which has the following asymptotics as $t \rightarrow-\infty$

$$
f \approx c(-t), c>0
$$

As a concrete example, we choose

$$
\begin{aligned}
& a_{2}(N)=-\frac{1}{N^{2}}+\frac{1}{3 N^{4}}, \\
& a_{3}(N)=\frac{1}{4 N^{3}} .
\end{aligned}
$$

The background metric reads

$$
d s^{2}=-N(t)^{2} d t^{2}+a(t)^{2} d x_{i} d x^{i}
$$

where $N$ is the lapse function (the same as in the Lagrangian (2)). One derives the equations of motion for the homogeneous background directly from the variation of the background part of the Lagrangian [24]

$$
\mathcal{L}^{(0)}=N a^{3}\left(A_{2}+2 A_{3} H+6 A_{4} H^{2}\right),
$$

(where $H=\dot{a} /(N a)$ ) and obtains

$$
\begin{aligned}
& \left(N A_{2}\right)_{N}+3 N A_{3 N} H+6 N^{2}\left(N^{-1} A_{4}\right)_{N} H^{2}=0 \\
& A_{2}-6 A_{4} H^{2}-\frac{1}{N} \frac{d}{d t}\left(A_{3}+4 A_{4} H\right)=0
\end{aligned}
$$


From these equations we find an asymptotic solution at early times $(t \rightarrow-\infty)$

$$
\begin{gathered}
H \approx \frac{\chi}{(-t)^{1+\delta}}, \\
a \approx 1+\frac{\chi}{\delta(-t)^{\delta}}, \quad N \approx 1,
\end{gathered}
$$

where $\chi$ is the combination of the parameters of the Lagrangian

$$
\chi=\frac{\frac{2}{3} M_{P l}^{2}+\frac{c}{4}(2 \alpha+1+\delta) M_{P l}}{4(2 \alpha+1+\delta) c^{2+\delta}} .
$$

An important feature of this solution is that

$$
B_{4}(t, N), A_{4}(t, N) \rightarrow 0 \quad \text { as } \quad t \rightarrow-\infty,
$$

and hence

$$
G_{4}(\phi, X) \rightarrow 0 \quad \text { as } \quad t \rightarrow-\infty .
$$

On the one hand, these are necessary conditions to avoid ghost or gradient instabilities during subsequent evolution [20]. On the other hand, Eqs. (19) and (20) signalize that the strong coupling energy scale in this theory tends to zero as $t \rightarrow-\infty$. The purpose of this paper is to see whether or not the latter feature spoils the classical field theory description of the early time evoltution, $t \rightarrow-\infty$.

\section{Strong coupling scale for perturbations versus classical scale}

We now consider the perturbations about the classical solution, and, for technical reasons, study scalar perturbations only. We comment on tensor sector later on. The perturbed metric for scalar sector has the following form

$$
d s^{2}=-N^{2} d t^{2}+\gamma_{i j}\left(d x^{i}+N^{i} d t\right)\left(d x^{j}+N^{j} d t\right)
$$

where

$$
N=1+\alpha, \quad N_{i}=\partial_{i} \beta, \quad \gamma_{i j}=a^{2} e^{2 \zeta} \delta_{i j},
$$

and $\alpha, \beta, \zeta$ are scalar perturbations. Expanding the action up to the second order, one obtains the following expression for the quadratic action in the unitary gauge

$$
\begin{aligned}
S_{\alpha, \beta, \zeta}^{(2)}=\int N d t a d^{3} x & {\left[-3 g_{\zeta}\left(\frac{a}{N} \dot{\zeta}\right)^{2}+c_{\zeta}(\partial \zeta)^{2}-3 a^{2} H^{2} m_{\alpha} \alpha^{2}+2 g_{\zeta} \partial \alpha \partial \zeta+6 \frac{a^{2}}{N} H f_{\alpha} \alpha \dot{\zeta}\right.} \\
+ & \left.2 \frac{a}{N} g_{\zeta} \zeta \partial^{2} \beta-2 a H f_{\alpha} \alpha \partial^{2} \beta\right],
\end{aligned}
$$

where $\partial$ denotes spatial derivatives, and

$$
\begin{aligned}
g_{\zeta} & =2\left(B_{4}+N B_{4 N}\right) \\
c_{\zeta} & =2 B_{4} \\
f_{\alpha} & =2\left(\frac{N A_{3 N}}{4 H}+B_{4}-N B_{4 N}-N^{2} B_{4 N N}\right), \\
m_{\alpha} & =B_{4}-N B_{4 N}+2 N^{2} B_{4 N N}+N^{3} B_{4 N N N} \\
& -\frac{1}{6 H^{2}}\left(A_{2}+3 N A_{2 N}+N^{2} A_{2 N N}\right)-\frac{1}{2 H}\left(N A_{3 N}+N^{2} A_{3 N N}\right) .
\end{aligned}
$$


Early-time asymptotics of the latter coefficients are

$$
\begin{aligned}
& g_{\zeta} \sim c_{\zeta} \sim(-t)^{-2 \alpha}, \\
& f_{\alpha} \sim(-t)^{-2 \alpha}, \\
& m_{\alpha} \sim-(-t)^{-2 \alpha+\delta} .
\end{aligned}
$$

Fields $\alpha$ and $\beta$ are constraint variables. One finds them by solving the constraint equations and plugs them back into the action. In this way one obtains the following expression for the unconstrained quadratic action

$$
S_{\zeta}^{(2)}=\int N d t a d^{3} x\left(\frac{\epsilon_{s}}{c_{s}^{2}} \frac{a^{2}}{N^{2}} \dot{\zeta}^{2}-\epsilon_{s}(\partial \zeta)^{2}\right),
$$

where

$$
\epsilon_{s}=\frac{1}{a N} \frac{d}{d t}\left(\frac{a g_{\zeta}^{2}}{H f_{\alpha}}\right)-c_{\zeta}, \quad c_{s}^{2}=\frac{\epsilon_{s}}{3 g_{\zeta}}\left(1-\frac{g_{\zeta} m_{\alpha}}{f_{\alpha}^{2}}\right)^{-1} .
$$

The asymptotics of the functions $\epsilon_{s}$ and $c_{s}$ are found from (25):

$$
\epsilon_{s} \sim(-t)^{-2 \alpha+\delta}, \quad c_{s}^{2} \sim(-t)^{0} .
$$

Since $2 \alpha-\delta>1$, see (9), the overall coefficient $\epsilon_{s}$ tends to zero as $t \rightarrow-\infty$, signaling the low strong coupling energy scale at early times.

In our dimensional analysis below we naturally use the canonically normalized field $\pi$ instead of $\zeta$. Since $a(t), N(t)$ and $c_{s}^{2}(t)$ tend to constants as $t \rightarrow-\infty$, the canonically normalized field is (modulo a constant factor)

$$
\pi=\sqrt{\epsilon_{s}} \zeta \propto|t|^{-\alpha+\delta / 2} \zeta
$$

The fact that the coefficient here tends to zero as $t \rightarrow-\infty$ is crucial for what follows.

To figure out the strong coupling scale in the scalar sector, we have to go one step further and consider cubic action. We use the results presented in [28] for cubic action for all of the scalar perturbations $\alpha, \beta$ and $\zeta$, solve the constraints in terms of $\alpha$ and $\beta$ and obtain the following expression for unconstrained cubic action:

$$
\begin{aligned}
S_{\zeta}^{(3)}= & \int N d t a d^{3} x\left\{\Lambda_{1}\left(\frac{a}{N} \dot{\zeta}\right)^{3}+\Lambda_{2}\left(\frac{a}{N} \dot{\zeta}\right)^{2} \zeta+\Lambda_{3}\left(\frac{a}{N} \dot{\zeta}\right)^{2} \partial^{2} \zeta+\Lambda_{4}\left(\frac{a}{N} \dot{\zeta}\right) \zeta \partial^{2} \zeta\right. \\
& +\Lambda_{5}\left(\frac{a}{N} \dot{\zeta}\right)\left(\partial_{i} \zeta\right)^{2}++\Lambda_{6} \zeta\left(\partial_{i} \zeta\right)^{2}+\Lambda_{7}\left(\frac{a}{N} \dot{\zeta}\right)\left(\partial^{2} \zeta\right)^{2}+\Lambda_{8} \zeta\left(\partial^{2} \zeta\right)^{2} \\
& +\Lambda_{9} \partial^{2} \zeta\left(\partial_{i} \zeta\right)^{2}+\Lambda_{10}\left(\frac{a}{N} \dot{\zeta}\right)\left(\partial_{i} \partial_{j} \zeta\right)^{2}+\Lambda_{11} \zeta\left(\partial_{i} \partial_{j} \zeta\right)^{2}+\Lambda_{12}\left(\frac{a}{N} \dot{\zeta}\right) \partial_{i} \zeta \partial^{i} \psi \\
& +\Lambda_{13} \partial^{2} \zeta \partial_{i} \zeta \partial^{i} \psi+\Lambda_{14} \partial^{2} \zeta\left(\partial_{i} \psi\right)^{2}+\Lambda_{15}\left(\frac{a}{N} \dot{\zeta}\right)\left(\partial_{i} \partial_{j} \psi\right)^{2}+\Lambda_{16} \zeta\left(\partial_{i} \partial_{j} \psi\right)^{2}+ \\
& \left.+\Lambda_{17}\left(\frac{a}{N} \dot{\zeta}\right) \partial_{i} \partial_{j} \zeta \partial^{i} \partial^{j} \psi+\Lambda_{18} \zeta \partial_{i} \partial_{j} \zeta \partial^{i} \partial^{j} \psi\right\},
\end{aligned}
$$

where $\psi=\partial^{-2}(a \dot{\zeta} / N) ; \Lambda_{1} \ldots \Lambda_{18}$ are functions of $g_{\zeta}, c_{\zeta} f_{\alpha}, m_{\alpha}, A_{2}, A_{3}, A_{4}, H$, and hence of time $t$. All of them have power-law behavior at early times $t \rightarrow-\infty$,

$$
\Lambda_{i} \sim(-t)^{x_{i}}
$$

where $x_{i}$ is a combination of $\alpha$ and $\delta$. 
For power couning purposes, every term $\mathbb{L}_{i}$ in the cubic Lagrangian $(i=1, \ldots, 18)$ can be schematically written as follows

$$
\mathbb{L}_{i}=\Lambda_{i} \cdot \zeta^{3} \cdot\left(\partial_{t}\right)^{a_{i}} \cdot(\partial)^{b_{i}}
$$

where $a_{i}$ and $b_{i}$ are the numbers of temporal and spatial derivatives, respectively. In terms of the canonically normalized field $\pi$ one has

$$
\mathbb{L}_{i}=\tilde{\Lambda}_{i} \cdot \pi^{3} \cdot\left(\partial_{t}\right)^{a_{i}} \cdot(\partial)^{b_{i}}
$$

where

$$
\tilde{\Lambda}_{i}=\Lambda_{i} \epsilon_{s}^{-3 / 2}=\Lambda_{i}|t|^{-\frac{3}{2}(\delta-2 \alpha)} \sim|t|^{x_{i}-\frac{3}{2}(\delta-2 \alpha)} .
$$

By naive dimensional analysis (dimension of $\left[\Lambda_{i}\right]=4-a-b,\left[\epsilon_{s}\right]=2$ ) we immediately find that the strong coupling energy scale associated with the term $\mathbb{L}_{i}$ is

$$
E_{\text {strong }}^{(i)} \sim \tilde{\Lambda}_{i}^{-\frac{1}{a_{i}+b_{i}-1}} \sim|t|^{-\frac{x_{i}+3 \alpha-3 \delta / 2}{a_{i}+b_{i}-1}}
$$

On the other hand, the inverse time scale of classical evolution is

$$
E_{\text {class }} \sim \frac{\dot{H}}{H} \sim|t|^{-1}
$$

Thus the condition for legitimacy of the classical treatment of the early evolution, $E_{\text {class }} \ll$ $E_{\text {strong }}^{(i)}$ for all $i$ reads

$$
x_{i}+3 \alpha-\frac{3}{2} \delta<a_{i}+b_{i}-1, \quad i=1, \ldots, 18
$$

Clearly, the most dangerous terms are those with the smallest combination $a_{i}+b_{i}-x_{i}$. By inspecting the behavior of $\Lambda_{i}$ one finds that this combination is the smallest for $i=1$ (given the constraints (9)), when

$$
\Lambda_{1} \sim(-t)^{1-2 \alpha+3 \delta}, \quad a_{1}=3, \quad b_{1}=0, \quad a_{1}+b_{1}-x_{1}=2+2 \alpha-3 \delta
$$

(as an example, the next term has $\Lambda_{2} \sim(-t)^{-2 \alpha+2 \delta}, a_{2}=2, b_{2}=0$ and $a_{2}+b_{2}-x_{2}=2+2 \alpha-2 \delta$; recall that $\delta>0$ ). Thus, the strong coupling regime can be avoided for $2 \alpha<2-3 \delta$, which together with (9) gives

$$
0<\delta<\frac{1}{4}, \quad 2-3 \delta>2 \alpha>1+\delta
$$

We conclude that the strong coupling regime is avoided (at least as far as the scalar perturbations are concerned), in the sense that the evolution remains classical at early times, provided one chooses the Lagrangian parameters $\alpha$ and $\delta$ in the dark grey allowed region shown in Fig. 1.

To get more confidence in the classical field theory treatment avoiding the strong coupling problem, one has to analyze tensor, tensor-tensor-scalar and scalar-scalar-tensor sectors of perturbations. It is likely, though, that they give weaker constraints than those presented above. 


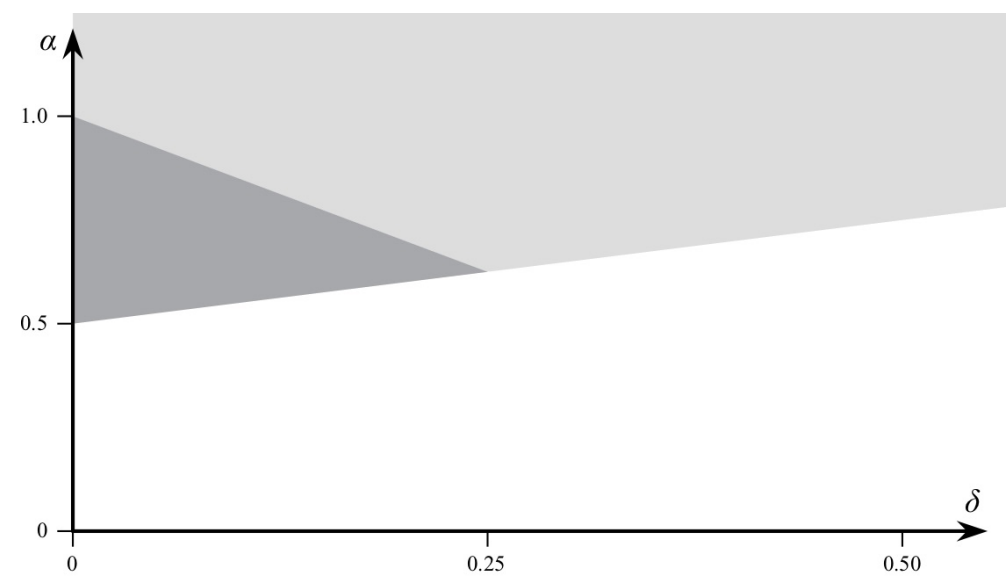

Figure 1. Light grey region corresponds to the Lagrangian parameters which yield Minkowski spacetime at $t \rightarrow-\infty$ and avoid the no-go theorem of Ref. [20]. Dark grey region shows the allowed range of the Lagrangian parameters satisfying "no strong coupling" criterion.

\section{Summary}

We have studied non-singular Genesis scenario in the framework of Horndeski theory, which is capable of avoiding the gradient instability at the expence of potential strong coupling problem. The model of Ref. [20] has been used as an example giving explicit asymptotic solutions at early times. We have seen that with appropriate choice of parameters, these solutions are actually away from the strong coupling regime inferred from the study of scalar perturbations. This opens up a posibility that the Universe starts up with very low quantum gravity energy scale (effective Planck mass asymptotically vanishes as $t \rightarrow-\infty$ ), and yet its classical evolution is so slow that the classical field theory description remains valid.

Even though our analysis has given promising outcome, it is certainly incomplete. First, we still have to study tensor perturbations and their cubic self-interactions and interactions with scalar perturbations. Second, there is no guarantee that the fourth and higher order interactions give strong coupling energy scales higher or equal to the ones we have found by studying the cubic interactions. We hope to turn to these issues in future.

\section{Acknowledgments}

We would like to thank E. Babichev, S. Mironov, A. Vikman and V. Volkova for numerous helpful discussions. This work has been supported by Russian Science Foundation grant 14-22-00161.

\section{References}

[1] P. Creminelli, A. Nicolis and E. Trincherini, JCAP 1011021 (2010).

[2] P. Creminelli, K. Hinterbichler, J. Khoury, A. Nicolis and E. Trincherini, JHEP 1302006 (2013).

[3] K. Hinterbichler, A. Joyce, J. Khoury and G. E. J. Miller, JCAP 1212030 (2012).

[4] K. Hinterbichler, A. Joyce, J. Khoury and G. E. J. Miller, Phys. Rev. Lett. 110, no. 24, 241303 (2013). 
[5] D. A. Easson, I. Sawicki and A. Vikman, JCAP 1307, 014 (2013).

[6] S. Nishi and T. Kobayashi, JCAP 1503, no. 03, 057 (2015).

[7] S. Nishi and T. Kobayashi, JCAP 1604, no. 04, 018 (2016).

[8] V. A. Rubakov, Phys. Usp. 57128 (2014).

[9] C. Armendariz-Picon, T. Damour and V. F. Mukhanov, Phys. Lett. B 458, 209 (1999).

[10] J. Garriga and V. F. Mukhanov, Phys. Lett. B 458, 219 (1999).

[11] G. W. Horndeski, Int. J. Theor. Phys. 10, 363 (1974).

[12] D. B. Fairlie, J. Govaerts and A. Morozov, Nucl. Phys. B 373214 (1992);

D. B. Fairlie and J. Govaerts, Phys. Lett. B 28149 (1992);

D. B. Fairlie and J. Govaerts, J. Math. Phys. 333543 (1992).

[13] M. A. Luty, M. Porrati and R. Rattazzi, JHEP 0309029 (2003);

A. Nicolis and R. Rattazzi, JHEP 0406059 (2004).

[14] A. Nicolis, R. Rattazzi and E. Trincherini, Phys. Rev. D 79064036 (2009).

[15] C. Deffayet, S. Deser and G. Esposito-Farese, Phys. Rev. D 82061501 (2010).

[16] C. Deffayet, O. Pujolas, I. Sawicki and A. Vikman, JCAP 1010026 (2010).

[17] T. Kobayashi, M. Yamaguchi and J. 'i. Yokoyama, Phys. Rev. Lett. 105231302 (2010).

[18] A. Padilla and V. Sivanesan, JHEP 1304032 (2013).

[19] M. Libanov, S. Mironov and V. Rubakov, JCAP 1608 no.08, 037 (2016).

[20] T. Kobayashi, Phys. Rev. D 94043511 (2016).

[21] R. Kolevatov and S. Mironov, Phys. Rev. D 94, no. 12, 123516 (2016).

[22] S. Akama and T. Kobayashi, Phys. Rev. D 95, no. 6, 064011 (2017).

[23] A. Ijjas and P. J. Steinhardt, Phys. Lett. B 764, 289 (2017).

[24] T. Kobayashi, M. Yamaguchi and J. Yokoyama, JCAP 1507 no. 07, 017 (2015).

[25] S. Nishi and T. Kobayashi, Phys. Rev. D 95064001 (2017).

[26] J. Gleyzes, D. Langlois, F. Piazza and F. Vernizzi, JCAP 1308025 (2013);

J. Gleyzes, D. Langlois, F. Piazza and F. Vernizzi, Phys. Rev. Lett. 114, no. 21, 211101 (2015).

[27] M. Fasiello, S. Renaux-Petel, JCAP 1410 no. 10, 037 (2014).

[28] X. Gao, D. Steer, JCAP 12019 (2011). 